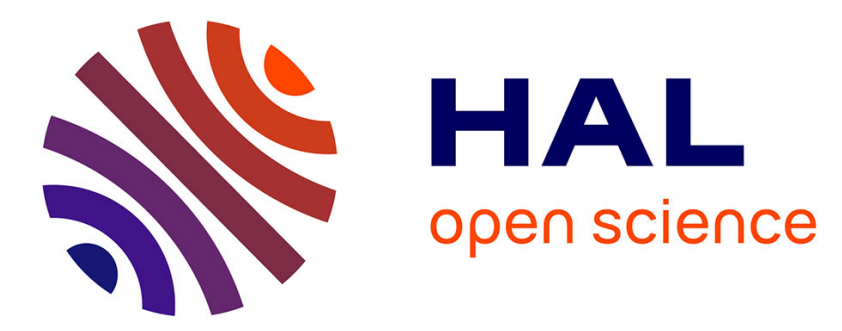

\title{
Plasma q -profile control in tokamaks using a damping assignment passivity-based approach
}

Ngoc Minh Trang Vu, Laurent Lefevre, Rémy Nouailletas, Federico Felici

\section{To cite this version:}

Ngoc Minh Trang Vu, Laurent Lefevre, Rémy Nouailletas, Federico Felici. Plasma q -profile control in tokamaks using a damping assignment passivity-based approach. Control Engineering Practice, 2016, 54, pp.34-45. 10.1016/j.conengprac.2016.05.003 . hal-01624840

\section{HAL Id: hal-01624840 https://hal.univ-grenoble-alpes.fr/hal-01624840}

Submitted on 26 Oct 2017

HAL is a multi-disciplinary open access archive for the deposit and dissemination of scientific research documents, whether they are published or not. The documents may come from teaching and research institutions in France or abroad, or from public or private research centers.
L'archive ouverte pluridisciplinaire HAL, est destinée au dépôt et à la diffusion de documents scientifiques de niveau recherche, publiés ou non, émanant des établissements d'enseignement et de recherche français ou étrangers, des laboratoires publics ou privés. 


\title{
Plasma $q$ profile control in tokamaks using a damping assignment passivity-based approach
}

\author{
VU Ngoc Minh Trang ${ }^{\mathrm{a}}$, Rémy NOUAILLETAS ${ }^{\mathrm{a}}$, Laurent LEFÈVRE ${ }^{\mathrm{b}}$, Federico FELICI $^{\mathrm{c}}$ \\ ${ }^{a}$ CEA, IRFM, F-13108 Saint-Paul-Lez-Durance, France \\ ${ }^{b}$ Univ. Grenoble Alpes, LCIS, F-26902, Valence, France \\ ${ }^{c}$ TU Eindhoven, Mechanical Engineering, Control Systems Technology Group, P.O. Box 5135600 Eindhoven, The Netherlands
}

\begin{abstract}
The IDA-PBC based on PCH model for tokamak $q$ profile is investigated. Two scenarios are carried out. The first one is the resistive diffusion model for the magnetic poloidal flux. The second one is extended with the thermal diffusion. A feedforward control is used to ensure the compatibility with the actuator physical ability. An IDA-PBC feedback is proposed to improve the system stabilization and convergence speed. The controllers are validated in the simulation using RAPTOR code and tested in TCV tokamak, the result is analyzed and the followed discussion proposed the required improvement for the next experiments.
\end{abstract}

Keywords: Tokamak plasma control, distributed parameter systems, Port-Controlled Hamiltonian systems, IDA-PBC control

\section{Introduction}

A Tokamak is a facility constructed with the shape of a torus (or dough-nut) in which a plasma is magnetically confined and heated in order to produce nuclear fusion reactions (see Fig. 1.1 for a schematic view and the classical Wesson's monograph [22] for a large comprehensive reference textbook). It aims at producing energy from the controlled nuclear fusion reactions. However many challenges remain to prove the scientific feasibility of this goal and then to move towards a fully functional plant. A suitable control model for these plasma dynamics is then a success key in the fusion research. There are many different objectives in Tokamak plasma control $[19,2]$. One of them consists in handling the MHD (Magneto-HydroDynamics) instabilities and improve the plasma confinement, while maintaining some current, temperature and pressure density profiles. Hence the goal is to reach some specific non-uniform profiles of the 1D plasma safety factor $q$ profile (equivalent to the inverse of plasma current density), an important parameter for both plasma stability and performance.

In this context the 1D resistive diffusion equation for the magnetic flux in the plasma ([3, Chap.6]) is a commonly used control model. Readers could refer to [23] for investigations on this model for control purposes or to [18] for application to model-based predictive control. A similar

Email addresses: ngoc-minh-trang.vu@cea.fr (VU Ngoc Minh Trang), remy.nouailletas@cea.fr (Rémy NOUAILLETAS), laurent.lefevre@lcis.grenoble-inp.fr (Laurent LEFÈVRE), F.Felici@tue.nl (Federico FELICI)

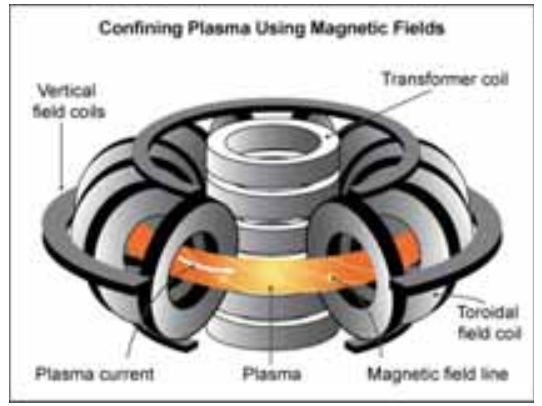

Figure 1.1: Schematic view of a tokamak with the electrical solenoids: the magnetic field generated by the three magnets makes the plasma gas ions following helicoidal trajectories along the torus

model has been used to solve the current profile optimal tracking problem [17] or to design robust controller for the poloidal magnetic flux profile in [16]. Feedback control using Lyapunov approach in [1], or sliding mode in [7] are also proposed. Note also that two-time scale extensions have already been considered for simultaneous magnetic and kinetic (temperature) profile control in tokamak [10].

This model also takes into account the plasma resistivity variations and the bootstrap current ${ }^{1}$ described in [22]. Both of these effects are large and very sensitive to the plasma temperature. When this dependence is considered, scaling laws are usually used to determine the system parameters (resistivity and bootstrap current), see [4] or [9]. However, to the best of our knowledge, there

\footnotetext{
${ }^{1}$ A magnetohydrodynamic coupling effect which produces an extra current density
} 
is no work proposing the design of feedback controls using both the plasma resistive diffusion equation and the plasma thermal equation (here roughly modeled using a heat transport equation), as well as the corresponding interdomain couplings and actuators in both magnetic and thermal domains to achieve a better safety factor profile regulation.

On the other hand, most of these models already possess an Hamiltonian structure which is considered essential by plasma physicists since the fundamental laws governing charged particle dynamics are Hamiltonian. Therefore the preservation of the Hamiltonian structure provides some confidences that the truncations used to derive the fluid model have not introduced unphysical phenomena. The presence of the Hamiltonian structure has the additional benefit of providing important tools for calculations such as the MHD energy principle, solvability conditions for the equilibrium equations, Casimir invariants, etc. A model based on a port-Controlled Hamiltonian (PCH) formulation of the plasma TMHD (Thermal-Magneto-HydroDynamics) in tokamaks is proposed in [21]. This model implies to modify the safety factor control problem into an equivalent magnetic field profile control problem. Spatial reduction and discretization methods, inspired from [11] and developed in [20], allow to reduce this 3D TMHD model to a finite dimensional PCH model. These symplectic reduction and discretization methods preserve the qualitative spectrum properties. Another consequence is that the finite-dimensional PCH model has the same invariants (for instance the total energy density) and model structure as the infinite-dimensional ones. Stored and dissipated energies in the finite dimensional model are simply approximation of the actual ones in the original distributed parameter model. Therefore, this finite-dimensional $\mathrm{PCH}$ model is the ideal one for the design of a high performance IDA-PBC (Interconnection and Damping Assignment - Passivity Based Control) controller [15]. This general control design, taking advantage of the Hamiltonian structured model, aims not only at sharping the total energy of the closed-loop system, but also at modifying the interconnection and dissipation structures of the original one. The controller achieves the robust stabilization by the passivity property of the desired closed loop Hamiltonian model.

Here, the proposed IDA-PBC controller allows stabilizing 1D profiles of the safety factor $q$ at the desired references directly by two actuators: the voltage $V_{\text {loop }}$ at the boundary of the plasma ${ }^{2}$ and the distributed non inductive current-drive heating source $J_{\text {ext }}$. Besides, a third actuator, the external heating source $S_{\text {heat }}{ }^{3}$, will be used as supplementary actuator which modifies the plasma tem-

\footnotetext{
${ }^{2}$ the loop voltage produced mainly by the central solenoid showed in figure 1.1

${ }^{3}$ The external current drive $J_{\text {ext }}$ and the external heating source $S_{\text {heat }}$ are both the effects from various antenna systems around the tore.
}

perature, hence indirectly some physical parameters such as the resistivity $\eta$ profile or the bootstrap current.

Challenges in this control problem arise not only from the time variation of some parameters usually badly estimated (such as the resistivity or diffusivity for instance), but also from the technological constraints and nonlinearities in the actuator models. In the considered facilities, the distributed controls $J_{\text {ext }}$ and $S_{\text {heat }}$ have specific spatial profiles, possibly depending from the control variable values themselves. In fact, the controllable inputs are rather the total external current power $P_{\text {ext }}$ and the total external heating power $P_{\text {heat }}$. The consequence is that the system is a finite rank input-output control system with both boundary and distributed control actions. The finite dimensional coupled control model may thus be considered as an under-actuated system in the sense that the number of actuators is less than the number of system states (more details on under-actuated PCH systems could be found in [13]). Hence, only a limited (finite dimensional) set of safety factor profiles are reachable. In this work, the available control signals are used to regulate the $q$-profile at a finite number of points. On one side, the corresponding $q$-profile on the whole spatial domain for the radial coordinate, as well as the corresponding feedforward control are both computed in order to guarantee their compatibilities with the systems constraints. On the other side, the designed IDA-PBC feedback control aims at improving the system stabilization and convergence rate as well as at attenuating the approximation errors. Nevertheless, an integrator is still necessary to cancel the static error on the safety factor profile.

Two scenarios are figured out in the sequel. In the first one the $\mathrm{PCH}$ model equivalent to the resistive diffusion equation is used with two control signals $V_{\text {loop }}$ and $P_{\text {ext }}$ to regulate the $q$ radial profile at two positions. In the second one, the magneto-hydro-dynamic couplings and the thermal-electromagnetic model are investigated. A third control signal $P_{\text {heat }}$ is used in order to reach a given reference value for the $q$ radial profile at a third point. The simulation results will be presented, they are based on the RAPTOR code (cf. [6, 5]) for the TCV (Tokamak of Variable Configuration at EPFL, Lausanne, Switzerland) tokamak real-time control system. Besides, based on these previous simulation tests, the IDA-PBC controller has also been implemented and tested on the real TCV experimental facility.

This paper is organized as follows. In section 2 , the IDA-PBC design methodology is revisited and adapted to the specific studied case. In section 3, the model plant is clearly explained, the control problem is reveals and the solution is proposed. The resistive diffusion model (cf. [20]) is firstly used as a control model in section 4. A non-linear feedforward control takes into account the system constraints and a simple "linear" IDA-PBC feedback control is discussed with the help of some practical considerations. Some simulation and experimental results are also figured out. In section 5, the coupled TMHD system 
(cf. [21]) is then used as a control model for the IDA-PBC controller design. The same methodology is adapted for new coupled system in 5.2 and the control law is tested only on simulation and compared to the previous one in subsection 5.4. The paper ends with a brief conclusion and some prospects for the future works.

\section{IDA-PBC closed loop control for PCH systems}

The IDA-PBC control design may be considered as the the most general one among passivity based control (PBC) designs for PCH systems. Readers may refer to [14] for an overview and connections between IDA-PBC controls and more particular types of PBC, such as energy shaping, power shaping, or control by interconnection. A brief reminder of the IDA-PBC design methodology (inspired from [15]) is given hereafter.

\subsection{Methodology overview}

Given a standard PCH system:

$$
\dot{x}=[\mathcal{J}(x)-\mathcal{R}(x)] \frac{\partial \mathbb{H}(x)}{\partial x}+g(x) u
$$

where $x \in \mathbb{R}^{n}$ is system state, $\mathcal{J}(x)=-\mathcal{J}^{T}(x)$ is a skewsymmetric interconnection matrix, $\mathcal{R}(x)=\mathcal{R}^{T}(x) \geqslant 0$ is a symmetric positive semi-definite dissipation matrix, $\mathbb{H}(x)$ is the total energy function or Hamiltonian and $g(x)$ is the control matrix which depends on system state $x$.

The main idea of the IDA-PBC method is to choose an appropriate feedback control law $u(x)$ so that the original system (2.1) is pulled back to a reference system with a set of desired properties. Let us design a closed loop reference system:

$$
\dot{x}=\left[\mathcal{J}_{d}(x)-\mathcal{R}_{d}(x)\right] \frac{\partial \mathbb{H}_{d}}{\partial x}(x)
$$

with $\mathcal{J}_{d}(x)=-\mathcal{J}_{d}^{T}(x), \mathcal{R}_{d}(x)=\mathcal{R}_{d}^{T}(x) \geqslant 0$ and a strict local minimum $x_{d}$ for the closed loop Hamiltonian $\mathbb{H}_{d}$. This minimum $x_{d}$ is a locally stable equilibrium since:

$$
\frac{d}{d t} \mathbb{H}_{d}=-\left(\partial_{x} \mathbb{H}_{d}\right)^{T} \mathcal{R}_{d}\left(\partial_{x} \mathbb{H}_{d}\right) \leq 0
$$

thanks to $\mathcal{J}_{d}(x)=-\mathcal{J}_{d}^{T}(x)$. The static state feedback is then chosen such that the closed-loop system matches this reference $\mathrm{PCH}$ system by using the "tuning parameters" $\mathcal{J}_{a}(x), \mathcal{R}_{a}(x), \mathbb{H}_{a}(x)$ such that $\mathcal{J}_{d}(x)=\mathcal{J}(x)+\mathcal{J}_{a}(x)$, $\mathcal{R}_{d}(x)=\mathcal{R}(x)+\mathcal{R}_{a}(x)$ and such that $\mathbb{H}_{d}(x)=\mathbb{H}(x)+$ $\mathbb{H}_{a}(x)$ has the minimum at $x_{d}$. This leads to a matching equation for the equivalence of (2.1) and (2.2) which reads:

$$
(\mathcal{J}-\mathcal{R}) \frac{\partial \mathbb{H}}{\partial x}+g u=\left(\mathcal{J}_{d}-\mathcal{R}_{d}\right) \frac{\partial \mathbb{H}_{d}}{\partial x}
$$

and leads to the feedback:

$$
u=\left(g^{T} g\right)^{-1} g^{T}\left[\left(\mathcal{J}_{d}-\mathcal{R}_{d}\right) \frac{\partial \mathbb{H}_{d}}{\partial x}-(\mathcal{J}-\mathcal{R}) \frac{\partial \mathbb{H}}{\partial x}\right]
$$

Besides, the following conditions are required for the solution:

i) (Integrability)

$$
\frac{\partial^{2} \mathbb{H}_{d}}{\partial x^{2}}(x)=\left[\frac{\partial^{2} \mathbb{H}_{d}}{\partial x^{2}}(x)\right]^{T}
$$

ii) (Equilibrium assignment)

$$
\frac{\partial \mathbb{H}_{d}}{\partial x}\left(x_{d}\right)=0
$$

iii) (Lyapunov stability)

$$
\frac{\partial^{2} \mathbb{H}_{d}}{\partial x^{2}}\left(x_{d}\right)>0
$$

The first condition implies the existence of the scalar energy function $\mathbb{H}_{d}$, while the two others ensure respectively the existence of the minimum of $\mathbb{H}_{d}$ at the desired equilibrium value $x_{d}$ and its stability.

This general design methodology preserves many degrees of freedom since the controller is set only once $\mathcal{J}_{a}, \mathcal{R}_{a}$ and $\mathbb{H}_{a}$ have been chosen. In this paper, we propose a particular design methodology for our system in the next subsections.

\subsection{Integrator extension}

An integral action may be added to the IDA-PBC control action in order to eliminate the static error due to the approximations or disturbances, while always conserving the PCH structure of the whole closed-loop system (a simple design for this integral action is proposed in $[12$, sec.6]). In this work, we have adapted this integrator into our system.

Proposition 2.1. Consider the system of equation (2.1) in closed-loop with $u=\beta(x)+v$ :

$$
\left[\begin{array}{c}
\dot{x} \\
\dot{x_{I}}
\end{array}\right]=\left[\begin{array}{cc}
\mathcal{J}_{d}(x)-\mathcal{R}_{d}(x) & K_{I} \\
-K_{I}^{T} & 0
\end{array}\right]\left[\begin{array}{c}
\partial_{x} W \\
\partial_{x_{I}} W
\end{array}\right]
$$

where $x_{I} \in \mathbb{R}^{k}$ are the extended integral variables and $K_{I} \in \mathbb{R}^{n \times k}$ the corresponding integral gains. The extended Hamiltonian

$$
W\left(x, x_{I}\right) \triangleq \mathbb{H}_{d}(x)+\mathbb{H}_{I}\left(x_{I}\right)
$$

qualifies as a new Lyapunov function, in which $\mathbb{H}_{I}$ is considered as the energy function associated with the integral effect. It may be designed as the usual quadratic form:

$$
\mathbb{H}_{I}=\frac{1}{2} x_{I}^{T} \mathcal{Q}_{I} x_{I} \quad \mathcal{Q}_{I}=Q_{I}^{T} \in \mathbb{R}_{+}^{k \times k}
$$

The integral contribution in the control action is then derived:

$$
v=-\left(g^{T} g\right)^{-1} g^{T} K_{I} \mathcal{Q}_{I} \int_{0}^{t} K_{I}^{T} \partial_{x} \mathbb{H}_{d}
$$

Stability properties of $x_{d}$ remain preserved since the augmented system (2.9) keeps the canonical PCH form. 
Remark 2.2. The IDA-PBC control law developed in the paper can be considered equivalent to a proportional controller. However, the controller gain is derived based on the system structure, which can guarantee the stability and the robustness against the system uncertainties and disturbances. The integrator is one of the extensions for $\mathrm{PCH}$ system which were presented in $[12$, sec.6]. The key point is that this integrator can be put around the passive output to preserve not only the stability, but also the $\mathrm{PCH}$ structure.

\section{Control problem and strategy}

The simplified tokamak plasma model in figure 1.1 is a multi-physics system, which can be represented in the figure 3.1. The governed equations are the Maxwell's equations in the electromagnetic domain, as well as the energy balance equations in the material domain. For the sake of simplicity, this work takes into account the quasi-static equilibrium assumption (cf. [3, Chap. 6]), in which the plasma may be assumed to have reached a mechanical stationary profile at every instant considered for the heat or magnetic flux diffusion phenomena. Therefore, only the thermodynamics associated with the heat transport equation is considered in the material domain.
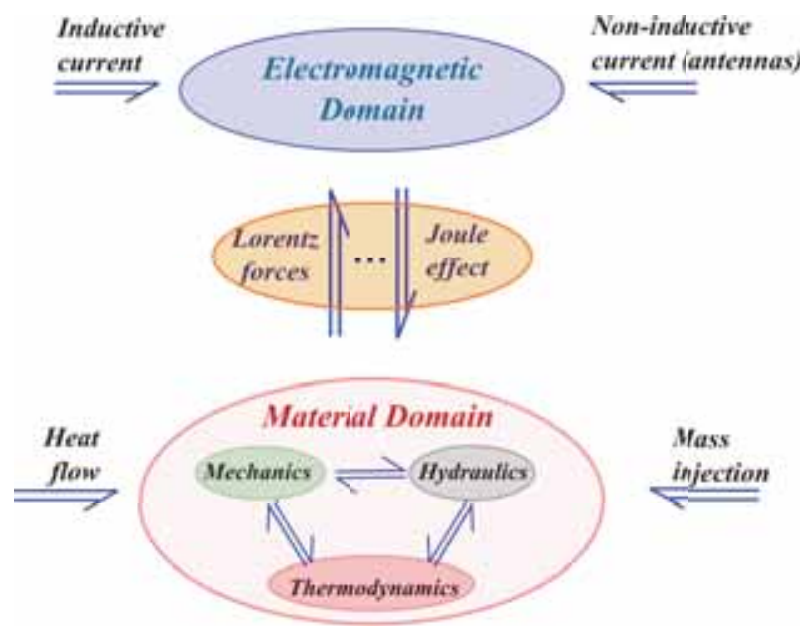

Figure 3.1: Multi-physics tokamak system

There are some proper actuators in each domain for plasma heating. The ohmic heating by inductive current is controlled by the boundary loop voltage $V_{\text {loop }}$ of the central solenoid (figure 1.1). However, when the temperature is over $1 \mathrm{keV}$, ohmic heating becomes practically useless due to the resistivity decreasing with the temperature. Hence, non inductive heating and current drive methods were developed to deal with this problem. The non-inductive current $J_{\text {ext }}$ and the heat flow $S_{\text {heat }}$ are both supplied by various antennas heating systems (the reader can refer to [22] for details of different antennas systems). The mass injection is used to control the plasma density by an independant system and not considered in this work. in this work.

Two strong couplings between the electromagnetic and thermal domains are the Lorentz force and the Joule effect. More precisely, these couplings are included in the system parameters, such as the resistivity $\eta$, the thermal diffusion coefficient $\chi$ and the bootstrap current $J_{b s}$, an extra current density produced by the magnetohydrodynamic coupling effect (cf. [22] ).

The paper is based on practical control issues on tokamak plasma, one of them is the advanced control of the safety factor profile $q$. This factor is in-fact the ratio between the magnetic toroidal field and the poloidal one, and it is approximated by inversion of the plasma current profile. The factor is really important for magnetohydrodynamic stability and plasma confinement; for example it is usually desirable $q>1$ (cf. [3, Chap. 6]) and to be kept the $q$-profile near the center as flat as possible. Sometime, the profile of $q<1$ is also studied for other plasma behaviors, such as the saw-teeth phenomenon (cf. $[22])$.

The plant model for the $q$ profile control is widely used as the resistive diffusion equation, which is a Partial Differential Equation (PDE) derived from the Maxwell's equations. The details of this controlled model are developed in section 4. Moreover, section 5 reveals a coupled controlled model to take into account the thermal domain with the heat transport equation and the system couplings for $q$ profile control, which makes the advantage of our control design in comparing with the previous control laws (model-based predictive control in [18], current profile optimal tracking in [17], robust controller for the poloidal magnetic flux profile in [16], Lyapunov approach in [1] or sliding mode in [7]) where only Maxwell's equations are considered.

The target profiles of $q$ are usually given by the physicists based much on the tokamak machine, accompany with the complex calculus programs as well as the test scenario.

In the sequel, the $q$ profile control using IDA-PBC method is tested with the RAPTOR code developed for the TCV tokamak (cf. $[6,5]$ ). RAPTOR is a $1 \mathrm{D}$ transport code specially designed for a fast execution compatible with the needs for real-time control or for nonlinear optimization schemes. The RAPTOR parameters are validated with experimental data. The code is also coupled to the real plant of TCV for some diagnostics and control purposes, such as giving some important plasma profile estimations: the $q$-profile, the resistivity $\eta$, the bootstrap current $j_{b s}$, and the control profile $f_{\text {ext }}$ or $f_{\text {heat }}$, etc. Hence, in our work, it is used to validate the control laws before implementing on TCV real plant.

\subsection{Control problem}

Besides the issue of the controller parameter choice $\left(\mathcal{J}_{a}, \mathcal{R}_{a}, \mathbb{H}_{a}\right)$ of the IDA-PBC method, the non-linearities 
and actuator constraints still complicate the control synthesis. Regarding the distributed controls, the real action signals are the total powers $P_{\text {ext }}(t)$ and $P_{\text {heat }}(t)$ of the external current drive $J_{\text {ext }}(z, t)$ and the external heating source $S_{\text {heat }}(z, t)$ respectively ( $z$ denotes the spatial coordinate). The control deposits $g(x) u(t)$ are approximated as:

$$
\begin{cases}J_{\text {ext }} & =f_{\text {ext }}(z, t) P_{\text {ext }}(t) \\ S_{\text {heat }} & =f_{\text {heat }}(z, t) P_{\text {heat }}(t)\end{cases}
$$

where $f_{\text {ext }}(z, t)$ and $f_{\text {heat }}(z, t)$ are the specific spatial deposit shapes of these "distributed" controls (typically Gaussian shapes), the control matrix $g(u)$ can be now considered spatially dependent $g(z)$.

These actuators are considered in the TCV case with ECCD ( Electron Cyclotron heating and Current Drive) system (cf. [23]). Two ECCD clusters ${ }^{4}$ are used to generate both the non-inductive current and the external heating source:

$$
\left\{\begin{array}{l}
P_{\text {ext }}=P_{A}-P_{B} \\
P_{\text {heat }}=\left(P_{A}+P_{B}\right)
\end{array}\right.
$$

The first one $P_{A}$ is used as a co-current source (to increase the total plasma current) and the second one $P_{B}$ as a counter-current source (to decrease the total plasma current).

Notice that in general, some external source deposit profiles also depend on the source total powers such that $f_{\text {ext }}:=f_{\text {ext }}\left(z, t, P_{\text {ext }}\right)$ and $f_{\text {heat }}:=f_{\text {heat }}\left(z, t, P_{\text {heat }}\right)$. One may refer to the Low hybrid heating source which is simply defined in [23] for more detail. Thus, when the non-linear control $g(x, u)$ does not correspond to the standard $\mathrm{PCH}$ form in (2.1), the simple feedback control derived in equation (2.5) can not be directly applied.

\subsection{Control strategy}

In order to deal with the system control problem, a feedforward ${ }^{5}$ control will be designed first which leads to a reachable steady state, then the closed-loop stabilization and the convergence rate will be improved via an IDAPBC feedback control. This (usual) control strategy is summarized in the figure 3.2 .

For the design of the IDA-PBC feedback control, in general, we will make use of the linearization of $g(x, u)$ around the equilibrium profile $(x, u)_{d}$ (where $u_{d}$ denotes the feedforward part of the control action which is designed with the non linear PCH model). This will help us to completely distinguish the feedforward and feedback effects, as well as allows us to apply the "traditional" IDA-PBC feedback design discussed in the previous subsection.

\footnotetext{
${ }^{4}$ The details for the TCV actuators may also be found in the website http://crpp.epfl.ch/research_TCV_Heating

${ }^{5}$ The term "feedforward" used in this paper refers to the stationary control at the equilibrium.
}

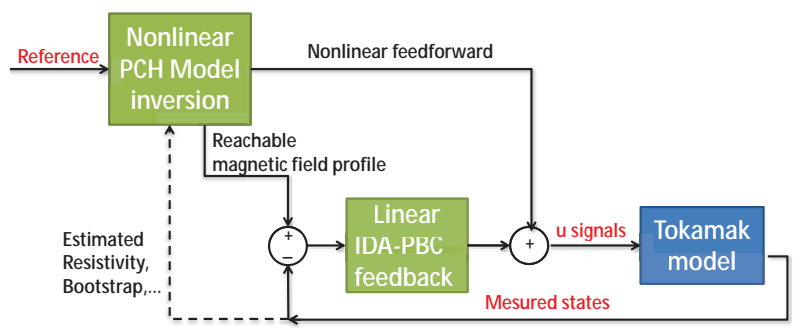

Figure 3.2: The proposed control strategy: a nonlinear feedforward control and a feedback control designed with the help of the linearized model

Proposition 3.1. Assuming a good accuracy of the model, the feedback effect contribution is considered widely weaker than the feedforward one into the total control law $g(x, u) u$. Thus the feedforward part of the controller is computed using non-linear expression of the system and a linearization around the equilibrium point can be done for the computation of the feedback part:

$$
\begin{aligned}
g(x, u) u & =g\left(x_{d}, u_{d}+\delta u\right)\left(u_{d}+\delta u\right) \\
& \approx g_{d} u_{d}+g_{d} \delta u
\end{aligned}
$$

where $g_{d}=g\left(x_{d}, u_{d}\right), u_{d}$ is the feedforward and $\delta u$ is a feedback control.

\subsection{Robustness analysis}

In this subsection, the robustness of the controller against two kinds of uncertainties is studied. The first one is on the system dissipation $\mathcal{R}$ coming from a bad estimation of the system parameters (such as the plasma resistivity $\eta$ and the thermal diffusion coefficient $\chi$ see [23]). The disturbance defined via the uncertainty on the resistivity $\delta \eta$ and on the thermal diffusion coefficient $\delta \chi$ leads to an uncertainty $\delta \mathcal{R}=\delta \mathcal{R}^{T}$ applied on $\mathcal{R}$. The disturbed system can be defined as:

$$
\dot{x}=\left[\mathcal{J}_{d}-\left(\mathcal{R}_{d}+\delta \mathcal{R}\right)\right] \partial_{x} \mathbb{H}_{d}
$$

With this kind of disturbance, the system is still stable as long as the total dissipation is positive, i.e. $\left[\mathcal{R}_{d}+\delta \mathcal{R}\right]>0$.

The second one concerns the uncertainty on the actuator profiles $\left(J_{\text {ext }}, S_{\text {heat }}\right.$ as well as a bad knowledge of the bootstrap current $\left.J_{b s}\right)$. It can also include the approximation error during the linearization in (3.3):

$$
g(x, u) u=g_{d} u_{d}+g_{d} \delta u+\underbrace{\delta g\left(u_{d}+\delta u\right)}_{\zeta}
$$

This kind of uncertainty is not trivial to stabilize by the choice of control parameters $\mathcal{R}_{d}$ and $\mathbb{H}_{d}$. This problem may be handled case-by-case by the small gain theorem (cf. [8]). 


\section{First case: the $\mathrm{PCH}$ resistive diffusion model}

The proposed IDA-PBC controller allows to stabilize the safety factor profile at the desired reference using two controlled (scalar) variables: the loop voltage $V_{\text {loop }}(t)$ and the external current power source $P_{\text {ext }}(t)$. The feedforward control is designed by choosing the (reachable) desired steady state. In this example, a simple controller tuning for the IDA-PBC parameters is proposed. This tuning is equivalent to damping assignment and energy shaping. The simulations chosen to illustrate the approach are based on TCV configuration.

\subsection{The resistive diffusion control model}

One obtains in [20], using our symplectic Galerkin scheme, the following time-dependent dissipative PortControlled Hamiltonian model equivalent to the resistive diffusion equation, derived from the Maxwell's equations in electromagnetic domain:

$$
\begin{aligned}
& \underbrace{\partial_{t}\left(\begin{array}{c}
\mathbf{d} \\
\mathbf{b}
\end{array}\right)}_{\partial_{t} x}=[\underbrace{\left(\begin{array}{cc}
0 & -J_{1} \\
-J_{2} & 0
\end{array}\right)}_{\mathcal{J}}-\underbrace{\left(\begin{array}{rr}
R^{-1}(z, t) & 0 \\
0 & 0
\end{array}\right)}_{\mathcal{R}}] \underbrace{\left(\begin{array}{cc}
G_{e l} & 0 \\
0 & G_{m g}
\end{array}\right)\left(\begin{array}{c}
\mathbf{d} \\
\mathbf{b}
\end{array}\right)}_{\partial_{x} \mathbb{H}_{E M}} \\
& +\underbrace{\left(\begin{array}{cc}
-f_{\text {ext }}-\frac{\mathrm{J}_{b s}}{P_{\text {ext }}} & 0 \\
J_{4}
\end{array}\right)}_{g(z)} \underbrace{\left(\begin{array}{c}
P_{\text {ext }} \\
V_{\text {loop }}
\end{array}\right)}_{u(t)}
\end{aligned}
$$

The bold variables $\mathbf{d}, \mathbf{b}, \mathbf{J}_{b s}$ and $\mathbf{J}_{\text {ext }}$ are the time-varying coefficients of the expansions in the chosen approximation bases of respectively the electric field, the magnetic field, the bootstrap current density and the external current source density. Note that the boundary control $V_{\text {loop }}(t)$ is now embedded in the finite dimension state equation. The matrices $J_{1}, J_{2}$ with $J_{1}=-J_{2}^{T}$ are obtained from the discretization (and reduction) of the spatial derivation $\partial_{z}$ in the effort approximation basis while $J_{4}$ is related to the boundary coefficient of this approximation. The dissipation matrix $R$ is determined by using the resistivity approximation in the same approximation bases $\eta(z, t)$. The total (approximate) energy $\mathbb{H}_{E M}$ is defined as a quadratic function:

$$
\mathbb{H}_{E M}=\frac{1}{2}\left(\mathbf{d}^{T} G_{e l} \mathbf{d}+\mathbf{b}^{T} G_{m g} \mathbf{b}\right)=\frac{1}{2} x^{T} \mathcal{Q} x
$$

where $\mathcal{Q}=\left(\begin{array}{cc}G_{e l} & 0 \\ 0 & G_{m g}\end{array}\right)$, the matrices $G_{e l}$ and $G_{m g}$ are symmetric positive and reduced to, respectively, the electric and magnetic permeability, $\frac{1}{\epsilon_{0} C_{3}}$ and $\frac{C_{2}}{\mu_{0}}$, in the simple anisotropic case. The coefficients $C_{2}, C_{3}$ are the toric coordinate coefficients defined by the plasma quasistatic equilibrium (cf. [3, Chap. 6]). The external current source is assumed equal to $\mathbf{J}_{\text {ext }}=f_{\text {ext }}(z) P_{\text {ext }}(t)$ where $f_{\text {ext }}(z)$ is the external current source spatial distribution.

This reduced system is thus defined directly in the usual explicit linear PCH form (2.1). Nevertheless, since the matrix $g(z)$ is not full rank, we have here an under-actuated system to deal with. Therefore, a reachable equilibrium as well as the corresponding feedforward is necessary to be determined in the sequel.

\subsection{Reference state generation for the resistive diffusion model}

The setting of the equilibrium point is restricted because of the actuator limitations previously discussed. To provide to the controller a reachable equilibrium profile, from two points of the reference $q_{\text {ref }}$ used by the physicist, the steady state $x_{d}=(\mathbf{d}, \mathbf{b})_{d}^{T}$ is computed (with $\mathbf{b}_{d}$ deduced from the inverse of the safety factor), and the corresponding feedforward control $u_{d}=\left(P_{\text {ext }}, V_{\text {loop }}\right)_{d}^{T}$ is derived. The plasma resistivity is supposed to be known as well as other system parameters. The equilibrium profiles $x_{d}$ will be adapted online to take into account the variations of the actuators parameters $f_{\text {ext.d }}$ and the reference $q_{\text {ref } f \text {. Using }}$ the geometric reduction and the symplectic discretization method in [20], the relation between the $q$-profile and the magnetic field $B_{\theta}$ (cf. [3, p.255]) becomes in the chosen approximation spaces:

$$
\bar{B}_{\theta}\left(z_{i}, t\right)=\mathbf{w}^{f}\left(z_{i}\right) \mathbf{b}_{d}(t)=2 \pi B_{\phi 0} a^{2} \frac{z_{i}}{q_{i}}
$$

where $q_{i}$ denotes the values of $q$ at the position $z_{i}, B_{\phi 0}$ the toroidal magnetic field intensity at $z=0, a$ is the small plasma radius and $\mathbf{w}^{f}\left(z_{i}\right)=\left(\begin{array}{lll}w_{1}^{f}\left(z_{i}\right) & \ldots & w_{N-1}^{f}\left(z_{i}\right)\end{array}\right)$ the $N-1$ Bessel approximation functions. On the other hand, at the equilibrium $\dot{x}_{d}=0$, the equation (4.1) becomes:

$$
\begin{aligned}
& \begin{cases}J_{1} G_{m g} \mathbf{b}_{d}+R^{-1} G_{e l} \mathbf{d}_{d}+\mathbf{J}_{\text {ext.d }}+\mathbf{J}_{\text {bs.d }} & =0 \\
-J_{2} G_{e l} \mathbf{d}_{d}+J_{4} V_{\text {loop.d }} & =0\end{cases}
\end{aligned}
$$

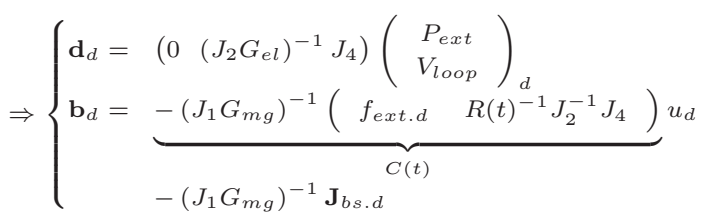

From (4.2) and (4.3), the feedforward control is therefore:

$$
\left(\begin{array}{c}
P_{\text {ext }} \\
V_{\text {loop }}
\end{array}\right)_{d}=\left(\left(\begin{array}{c}
\mathbf{w}^{f}\left(z_{1}\right) \\
\mathbf{w}^{f}\left(z_{2}\right)
\end{array}\right) C(t)\right)^{-1} \times 2 \pi\left(B_{\phi 0} a^{2}\right)\left(\begin{array}{c}
z_{1} / q_{1} \\
z_{2} / q_{2}
\end{array}\right)
$$

\subsection{IDA-PBC simple choice: energy shaping and damping assignment}

In the sequel, a non-linear "proportional" feedback control is proposed. The desired energy $\mathbb{H}_{d}=\mathbb{H}+\mathbb{H}_{a}$ is assumed to be the usual a quadratic form:

$$
\mathbb{H}_{d}=\frac{1}{2} X^{T} \mathcal{Q}_{d} X=\frac{1}{2} X^{T}\left(\mathcal{Q}+\mathcal{Q}_{a}\right) X
$$

with $X=x-x_{d}$ the state error and $\mathcal{Q}_{d}=\mathcal{Q}_{d}^{T}>0$ (in such a way that $\mathbb{H}_{d}$ satisfies the integrability, equilibrium and stability conditions (2.6-2.8)).

We propose hereafter a simple parameter tuning to modify only the damping and the energy of the system. We choose $\mathcal{J}_{a}=0$ since the interconnection structure $\mathcal{J}_{d}$ doesn't modify the convergence speed of the system total energy $\mathbb{H}_{d}$ in equation (2.3). The matrix $\mathcal{Q}_{a}$ shapes 
the total energy storage function $\mathbb{H}_{d}$ of the closed loop system, while $\mathcal{R}_{a}$ plays the role of adding dissipation. Since the initial system has dissipation $\mathcal{R}$ only on the electric field domain (linked to the first variable $\mathbf{d}$ ), it's possible to add dissipation on the other field (linked to the second magnetic domain whose state is described by the variable $\mathbf{b}$ ), via the matrix $\mathcal{R}_{a}$ by using the form $\mathcal{R}_{a}=\mathcal{R}_{a}^{T}=\left[\begin{array}{cc}0 & 0 \\ 0 & R_{a}\end{array}\right], 0 \leqslant R_{a} \in \mathbb{R}^{n \times n}$ where $R_{a}$ is chosen to be a diagonal matrix (only on the whole system state) or eventually only has some non zero diagonal values corresponding to the two reference positions $z_{1}$ and $z_{2}$. The higher the value of $\mathcal{R}_{a}$ is, the faster the system will converge. However, the input power limitation on the loop voltage $V_{\text {loop }}$ doesn't allow to set a huge value for $\mathcal{R}_{a}$.

The choice of $\mathcal{Q}_{a}$ is more complicated. The only condition to be satisfied is $\mathcal{Q}_{d}=\mathcal{Q}+\mathcal{Q}_{a}>0$. One can refer to section 3 in [15] for a detailed discussion about how to choose $\mathcal{Q}_{a}$ so-that the matching equation could be (fully or partially) satisfied. An arbitrary choice of $\mathcal{Q}_{a}$ will anyway give an equivalent control signal by applying (2.5). However, the control law in this case doesn't fulfill the matching equation (2.4). This also means that the static feedback transforms the original system (2.1) into the desired one (2.2) with an unknown error coming from the matching equation error. Thus, nothing guarantees the existence of a control law agreeing with a particular choice of IDA-PBC parameters.

One of the approximating solutions proposed in [15] is to pre-multiply the matching equation (4.6) with the left annihilation $g^{\perp}$ of $g_{d}$ (i.e. such that $g_{d}^{\perp} g_{d}=0$ ), and to choose $\mathcal{Q}_{a}$ as the solution of the "linear" equation:

$$
0=g_{d}^{\perp} g_{d} \delta u=g^{\perp}\left(\left[\mathcal{J}-\left(\mathcal{R}+\mathcal{R}_{a}\right)\right] \mathcal{Q}_{a} X-\mathcal{R}_{a} \mathcal{Q} X\right)
$$

One should remark that $\mathcal{Q}_{a}$ defined in this way is timedependent due to its dependence with $\mathcal{R}(z, t)$.

\subsection{Simulation results}

In the simulations, the electronic temperature profile of the plasma is computed using a thermal diffusion model in the RAPTOR/TCV. The plasma resistivity mainly depends on this temperature whereas the bootstrap current is a function of its gradient.

Only in this test scenario, the total heating source $P_{\text {heat }}$ is kept unchanged during the plasma discharge. We also consider that all the states are measurable or computable from measurements. One can "correctly" estimate the whole profile of $q$ in TCV. Two reference values for the safety factor $q$ are defined at the radial relative coordinates $z_{1}=0.1$ and $z_{2}=0.4$ which are in the interesting zone. The feedforward calculus gives $u_{d}$ as well as the reference profile $q_{d}$ (corresponding to the two reference values and taking into account actuator limits). The IDA-PBC control determines the feedback signal $\delta u$ from (3.3) to correct the error $X$.
Remark 4.1. In the TCV case, the output $q$-profile determined by RAPTOR is based on a finite element discretization method of the resistive diffusion model, while the one in our model comes from a symplectic geometric method ([20]). While the $q_{\text {ref }}$ is determined from our PCH model in (4.1). This explains the non-perfect match of two $q$ profiles showed in the figures 4.1-4.3. In other words, by increasing the number of approximation base functions, the error can be decreased and the complexity and the calculating time increase. The figure 4.4 illustrates the model with 20 base functions instead of 5 in the figure 4.3. The precision is much improved at the plasma edge and mostly unchanged at the center. The controller objective is however only to regulate $q$ at some chosen positions, usually near the center (and slightly relaxes elsewhere). Therefore, the approximation choice is a compromise between precision and complexity, especially regarding real-time control where the complexity is not promoted. Hence, the small number of base functions is used in the sequel.

In the test case, the reference $q$-profile is set with the values at the two points $z=0.1,0.4$ as $q_{a}=(0.85,1.25)$. The heating power is switched on at $t=0.2 \mathrm{~s}$, the feedback control starts at $t=0.45 \mathrm{~s}$. Then at $t=1.2 \mathrm{~s}$, the reference is changed to the new desired profile $q_{b}=(1,1.25)$. We denote error 1,2 the gaps between $q$ and $q_{\text {ref }}$ profiles at two considered positions.

Since the system is naturally dissipative, the feedforward in figure 4.1.a shows the convergence of the opened-loop system. Thanks to the re-computation of the equilibrium profile as in (4.3) at each step time, the $q$-profile reaches a steady state which is quite closed to the reference values. In figure 4.2 and 4.3 , the response time is decreased by the feedback effect via the $\mathcal{R}_{a}$ damping and the $\mathcal{Q}_{a}$ energy shaping.

It's important to note that, in general the feedback effect does not significantly acts on the considered particular positions. It does ameliorate the whole $q$ - profile when more damping assignment is added (see figure 4.2 and 4.3 ).
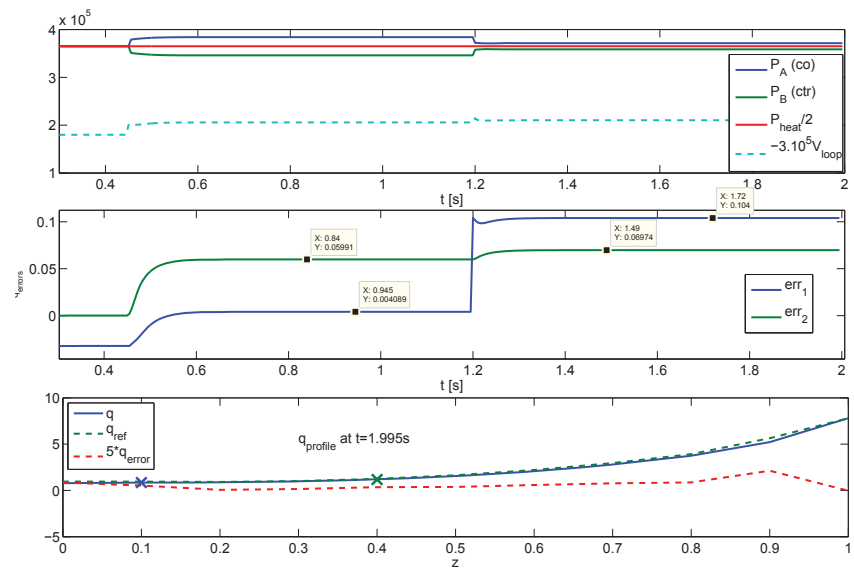

Figure 4.1: Open-loop response with feedforward control 

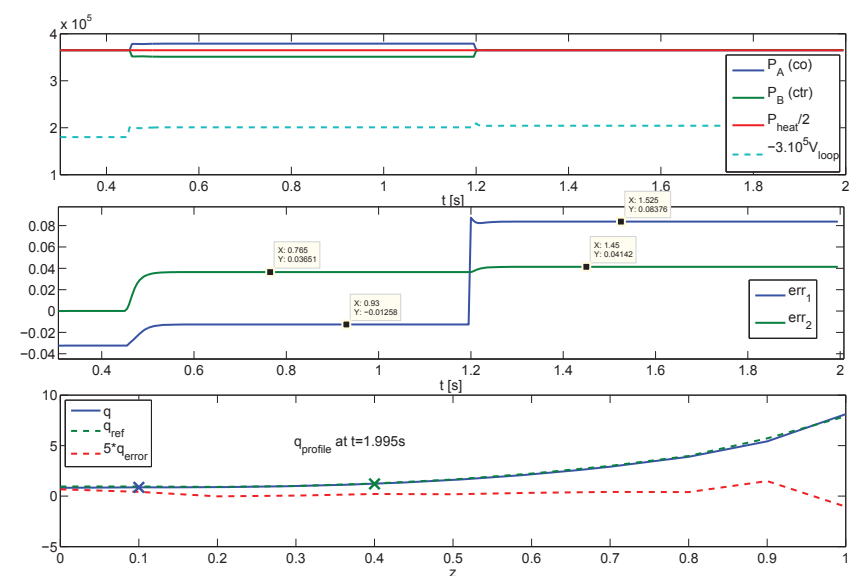

Figure 4.2: Closed loop system with an IDA-PBC controller and a small damping $\mathcal{R}_{a}$
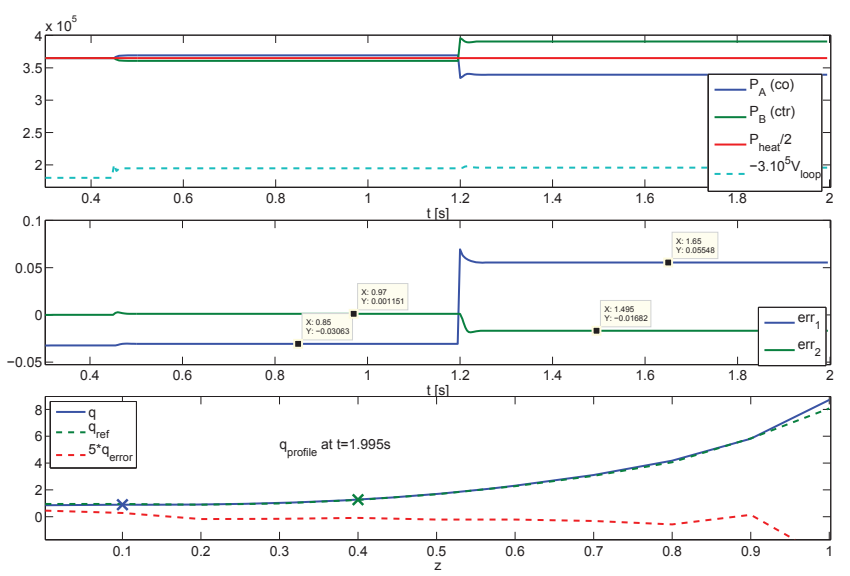

Figure 4.3: Closed loop system with an IDA-PBC controller and a larger damping $\mathcal{R}_{a}$
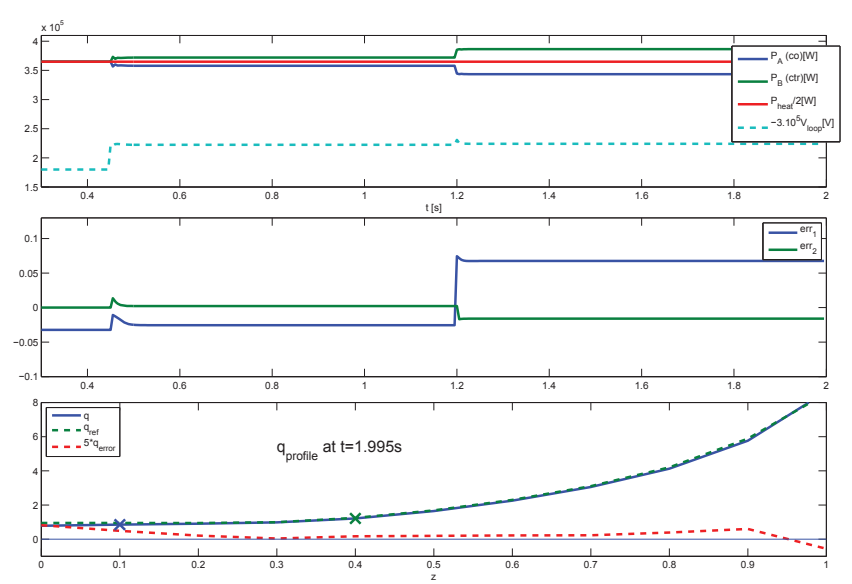

Figure 4.4: Simulation with 20 base functions and larger damping $\mathcal{R}_{a}$

Since there are always static errors caused by the approximations in the linearization (see subsection 3.2) of the actuator distribution profile and in the state estimation in (3.2), an integrator is added to the studied scenario and results are shown in figure 4.5.

Remark 4.2. The errors on the $q$-profile at the two considered positions are totally eliminated after $0.5 \mathrm{~s}$. However, it is "expensive" in term of controller energy to compensate the gap on the two values of the $q$-profile below 0.05. Furthermore, $P_{A}$ can't be technically set under the power of $150 K W$ and consequently the absolute convergence seen in figure 4.5 is unreachable in the reality. On the other hand, a small error on $q$-profile doesn't lead to a significant change in the behavior of the whole system.

In the figure 4.6, two disturbances are added. An error of $0.5 \eta$ is added on the measured resistivity at $t=0.65 \mathrm{~s}$ and an error of $0.5 f_{\text {ext }}$ is added on the estimated actuator profile at $t=0.9 \mathrm{~s}$. These perturbations are effectively rejected. As expected, the loop voltage $V_{\text {loop }}$ predominantly corrects the perturbation on resistivity while the external current source is used to compensate the distributed error on the current profile.

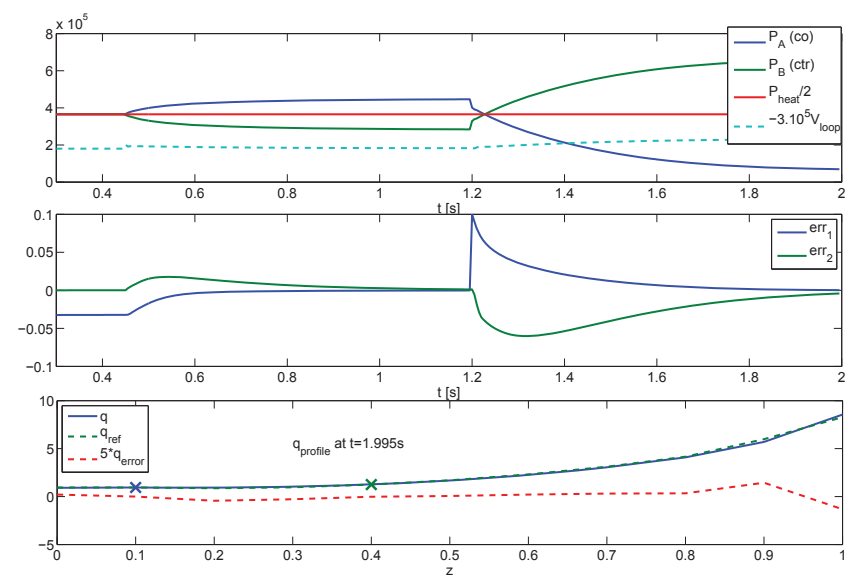

Figure 4.5: Closed loop system with IDA-PBC controller with integrator effect

\subsection{Experimental result}

The IDA-PBC control with the simple damping and energy shaping in subsection 4.3 was implemented on TCV Tokamak in its last campaign before its reconstruction and maintenance in 2013. The real experimental data are compared to the simulation one in the figure 4.7. The test scenario is set as the same as our previous simulation in the previous subsection.

The controller reacted as predicted in the first period around $0.45 s-0.8 s$. The average control values are consistent with the simulation result for all $P_{A}, P_{B}$, and $V_{\text {loop }}$. Unfortunately one of the cluster (the co-current source, cluster $A$ ) stopped working at $t=0.8 s$. From then we lost the control efficiency as the controller wasn't informed about this event. However, the closed loop control at least acts in the "good direction" (using cluster $B$ only) when 

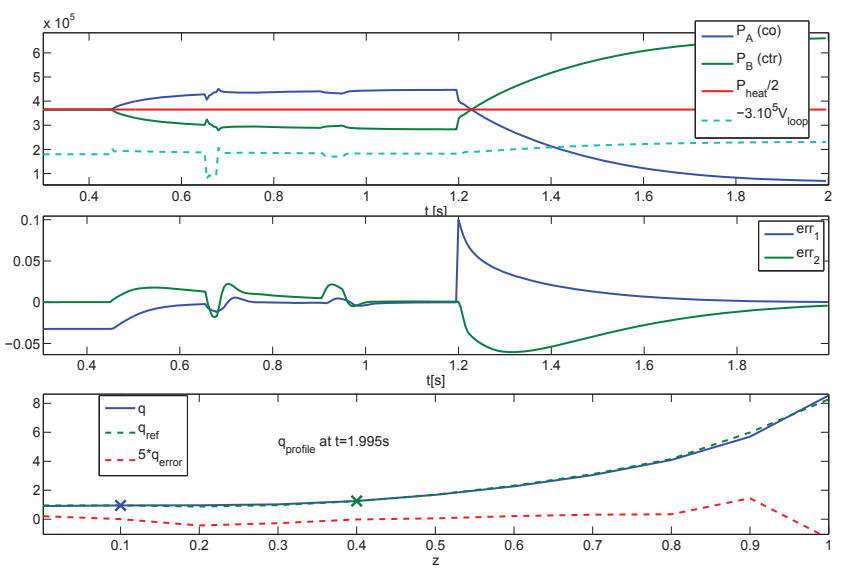

Figure 4.6: Closed loop system with IDA-PBC controller (with the integrator) with perturbations on the resistivity and on the actuator spatial distribution

$q_{\text {ref }}$ profile reference changed until the end. We can remark at $t=0.8 s$, when $P_{A}$ was cut off, the simulation realized the variation of $q$-profile, then reacted on $P_{B \text {.sim }}$, whilst in practice, it seemed that the controller didn't figure out the change. It maintained $P_{B}$ until the reference moved to the new profile. One of the unexplained observation is that the real controller $P_{B}$ responded more slowly than in simulation, even in comparing to the beginning of the feedback at $t=0.45 \mathrm{~s}$.

The $q$-profile at two considered positions are also showed in the figure 4.7. The feedback control is re-simulated based on the experimental average plasma density $\bar{n}$ from the shock 49514. The experimental control signals are equivalent to the simulated one, the figure 4.7 shows no differences but the measure noise between them. Furthermore, the measured values of the $q$-profiles do match quite well the simulation ones.

\section{Second case: TMHD Coupled system}

Actually, the resistive diffusion model used in the previous section is equivalent to the resistive diffusion equation which is widely used as a control model for the plasma (when one tries to regulate the safety factor profile, for instance). However, as the influence of the temperature $T$ on certain parameters in electromagnetic domain, such as the resistivity $\eta(T)$ and the bootstrap non-inductive current $J_{b t}\left(\partial_{z} T\right)$, is not negligible. The MHD couplings between the electromagnetic and thermal domains are presented in [21] in the irreversible entropy source term and in the bootstrap current source.

Therefore, a coupled control model made with the resistive diffusion submodel and the thermal diffusion submodel, is investigated hereafter. This finite dimensional control model is obtained with the coupling of the two finite dimensional approximations from the two diffusion models, using two geometric reduction schemes. Again, a
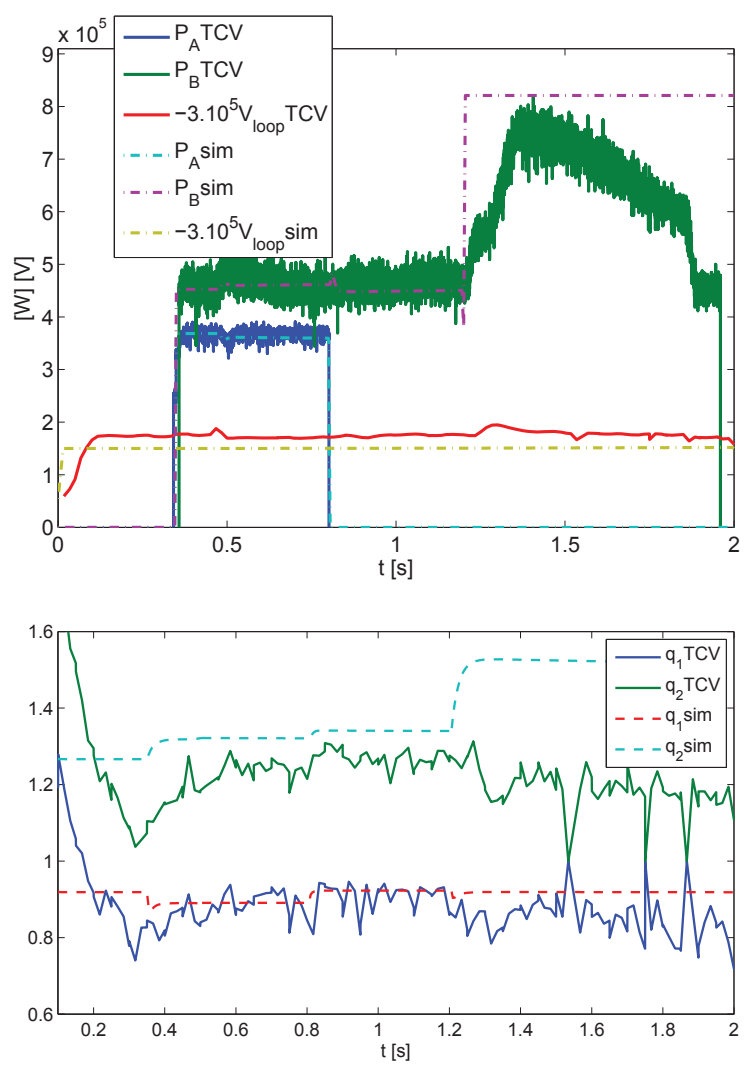

Figure 4.7: TCV shock 49514

feedforward control will be used to guarantee the compatibility of the designed control with the actuator constraints. Then, an IDA-PBC feedback law will be synthesized using the linearized error system.

As the result of adding the thermal diffusion submodel, a third actuator, the external heating power source $P_{\text {heat }}$, will be involved. Then, due to the under-actuation of the studied system, only the values of the safety factors at three reference points $z_{1}, z_{2}$ and $z_{3}$, denoted respectively $q_{1}, q_{2}$ and $q_{3}$ will be assigned with the help of the three available control variables $V_{\text {loop }}, P_{\text {ext }}$ and $P_{\text {heat }}$. However, the corresponding full radial profile reference for $q$-profile (hence for the state variables of our model) is required in order to define our IDA-PBC state feedback. This profile will be computed by taking into account at the same time the actuation constraints and the TMHD couplings. This procedure leads to an achievable steady state for the feedback design and, on the other hand, transforms the feedback design into a linearized IDA-PBC feedback control problem. The obtained numerical results are validated with the RAPTOR code.

\subsection{TMHD control system}

The first resistive diffusion submodel is recalled in equation (4.1) in the previous section. The second submodel is the discrete thermal model derived from the $1 \mathrm{D}$ portHamiltonian thermal diffusion model (cf. [21]), using the 
same discretization methodology as for the resistive diffusion submodel. This finite-dimensional approximation reads:

$$
\begin{gathered}
\left(\begin{array}{c}
\partial_{t} \mathbf{e}_{e x} \\
0
\end{array}\right)=\left[\left(\begin{array}{cc}
0 & J_{T 1} \\
J_{T 2} & 0
\end{array}\right)_{\bar{S}}-\left(\begin{array}{cc}
0 & 0 \\
0 & R_{T}^{-1}
\end{array}\right)\right]\left(\begin{array}{c}
\mathcal{Q}_{T} \mathbf{e}_{e x} \\
\mathbf{f}_{q}
\end{array}\right) \\
+\left(\begin{array}{c}
J_{T 4} T_{1}
\end{array}\right) \\
\mathbb{H}_{T}=\frac{1}{2} \mathbf{e}_{e x}^{T} \mathcal{Q}_{T} \mathbf{e}_{e x}
\end{gathered}
$$

where $\mathbf{e}_{e x}, \mathbf{f}_{q} \in \mathbb{R}^{N \times 1}$ are respectively the time dependent coefficients of $T D_{t} s$ and of the heat flux $f_{q}$. The matrices $J_{T 1}, J_{T 2} \in \mathbb{R}^{N \times N}$ with $J_{T 1}=-J_{T 2}^{T}$ are obtained from the reduction of the spatial derivation operator $\partial_{z}$ in the chosen finite dimensional spatial approximation bases. $\mathcal{Q}_{T} \in \mathbb{R}^{N \times N}$ is the positive definite matrix obtained from the discretization of the constitutive relation for the energy (i.e. between $e_{e x}$ and $T$ ) and $R_{T} \in \mathbb{R}^{N \times N}$ is the thermal resistivity approximation which depends on the thermal diffusion coefficient $\chi$. This thermal diffusivity coefficient (in our model) is used to write the dissipative constitutive relation between the thermal force $F$ and the heat flux $f_{q}$. $J_{T 4} \in \mathbb{R}^{N \times 1}$ is related to the boundary coefficient, $T_{1}$ is the fixed value of the average temperature at the boundary and $\mathbb{H}_{T}$ is the thermal energy.

Note that the PCH model for the thermal diffusion (5.1) is given in implicit form only, since there's only one balance equation for the entropy. The second equation (used to close the constitutive equation with the skew-adjoint operator to form the Dirac structure) is the dissipative relation giving the thermal force.

The aggregation of the resistive diffusion and the heat transport submodels results in the finite dimensional TMHD model which is written in the (implicit) PCH-like form with $x=\left(\mathbf{d}, \mathbf{b}, \mathbf{e}_{e x}\right)^{T}$ :

$$
\left(\begin{array}{c}
\dot{x} \\
0
\end{array}\right)=[\mathcal{J}(x)-\mathcal{R}(x)]\left(\begin{array}{c}
\partial_{x} \mathbb{H} \\
\mathbf{f}_{q}
\end{array}\right)(x)+g u
$$

where

$$
\begin{aligned}
& \mathcal{J}=-\mathcal{J}^{T}=\left[\begin{array}{cc}
-\left(\begin{array}{cc}
0 & J_{1} \\
J_{2} & 0
\end{array}\right) & \left.\begin{array}{cc}
0 \\
0 & J_{T 1} \\
0 &
\end{array}\right)
\end{array}\right]
\end{aligned}
$$

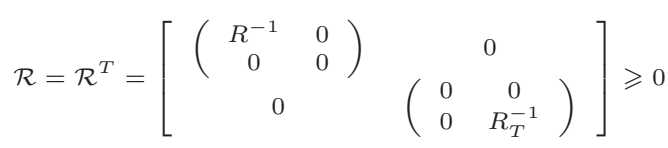

$\mathcal{J}$ is a skew-symmetric interconnection matrix defining a corresponding Dirac structure while $\mathcal{R}$ is a symmetric positive semi-definite dissipation matrix which is nonlinearly depending on the state variables. The total energy function or Hamiltonian is simply the sum of electromagnetic and thermal energy: $\mathbb{H}=\mathbb{H}_{E M}+\mathbb{H}_{T}$. As a consequence of this port-Hamiltonian representation for the TMHD model, the IDA-PBC approach for nonlinear control may be applied to the whole interconnected model as it was already the case for the electromagnetic part of it (equivalent to the resistive diffusion model).

The control of the interconnected system (resistive diffusion and thermal diffusion equations) is expected to take advantage of the explicit state space representation of the TMHD coupling analysis to improve the control performance through a better parameter estimations for the resistivity $R$ (via a good approximation of $\eta\left(T_{e}\right)$ and of the bootstrap current $J_{b s}\left(T_{e}, \partial_{z} T_{e}\right)$ ), as well as for the thermal resistivity $R_{T}$ (via the diffusion coefficient $\left.\chi\left(\partial_{z} T, B\right)\right)$.

Besides, the control actions are assumed to satisfy specific shapes (radial distribution) via the functions $f_{\text {ext }}$ and $f_{\text {heat }}$ (namely Gaussian distributions in the studied case). Therefore control variables are the scalar total external current power $P_{\text {ext }}$ and heating power $P_{\text {heat }}$. As discussed in subsection 3.2 , this implies that only a limited set of equilibrium states $x_{d}=\left(\mathbf{d}, \mathbf{b}, \mathbf{e}_{e x}\right)_{d}^{T}$ are reachable. Thus a feedforward control will be designed first which leads to a reachable steady state for which the closed loop convergence of the feedback diffusion system may be obtained via an IDA-PBC controller. Previously, a feedforward control $\left(P_{\text {ext }}, V_{\text {loop }}\right)_{d}^{T}$ has been proposed to achieve the regulation for two reference points of the safety factor profile: at the center and at the boundary. Here, using the interconnected TMHD model will allows us to add a third reference point of $q$ by the use of the new control action $P_{\text {heat }}$.

\subsection{Steady state generation for coupled TMHD model}

The steady state $x_{d}$ of (5.2) satisfies:

$$
\begin{cases}J_{1} G_{m g} \mathbf{b}_{d}+R^{-1} G_{e l} \mathbf{d}_{d}+f_{\text {ext }} P_{\text {ext }}+\mathbf{J}_{b s} & =0 \\ -J_{2} G_{e l} \mathbf{d}_{d}+J_{4} V_{\text {loop.d }} & =0 \\ J_{T 1} \mathbf{f}_{q d}+\bar{S} & =0 \\ J_{T 2} G_{T} \mathbf{e}_{e x}-R_{T}^{-1} \mathbf{f}_{q d}+J_{T 4} T_{1} & =0\end{cases}
$$

The following points could be noticed :

- The input signal $T_{1}$ is assumed constant in these equilibrium equations $\left(T_{1}=0\right.$ for instant)

- The source term $\bar{S}$ includes the Joule effect $S_{J o u l e}=$ $\eta J_{\text {tot }}\left(J_{t o t}-J_{n i}\right)$ (where $J_{t o t}$ is the total current density) and the external heating source $S_{\text {heat }}$ which is controlled by the heating power $P_{\text {heat }}$.

- The TMHD couplings - including the terms $J_{b s}\left(B_{\theta}, T, \partial_{z} T\right), \eta(T)$, and $\chi\left(\partial_{z} T, B_{\theta}\right)$ - are estimated by empirical analytic expressions given in [23]. With the assumption that the thermal steady state for $T$ and $\partial_{z} T$ are quickly established and also assuming a constant particle density $n$, we can deduce from these expressions the following values for:

- the resistivity coefficient :

$$
\eta(T)=C_{\eta}(\mathbf{b}) T^{-3 / 2}
$$


- the thermal diffusion coefficient:

$$
\chi\left(\partial_{z} T, B_{\theta}\right)=C_{\chi}(\mathbf{b}) \partial_{z} T
$$

- the discrete bootstrap current:

$$
\begin{aligned}
\mathbf{J}_{b s} & =\frac{1}{\mathbf{b}}\left(\beta_{1} \mathbf{e}_{e x}+\beta_{2} R_{T}^{-1} \mathbf{f}_{q}\right) \\
& =\frac{1}{\mathbf{b}}\left(\beta_{1}\left(J_{T 2} G_{T}\right)^{-1}+\beta_{2}\right)\left(J_{T 1} R_{T}\right)^{-1} \bar{S} \\
& =\frac{1}{\mathbf{b}}\left(\beta_{1}\left(J_{T 2} G_{T}\right)^{-1}+\beta_{2}\right)\left(J_{T 1} R_{T}\right)^{-1} \\
& \times\left(S_{\text {Joule }}+S_{\text {heat }}\right)
\end{aligned}
$$

where $C_{\eta}(\mathbf{b}), C_{\chi}(\mathbf{b}), \beta_{1}$ and $\beta_{2}$ are the coefficients depending on the system state (cf. [23]). The Joule effect $S_{\text {Joule }}$ may be considered as a measurable output assuming that the total current and the external non-inductive current are known. It's also one of the MHD couplings but in practice, it is negligible when compared to the external heating source. $S_{\text {heat }}$ is given by the analytic expression $f_{\text {heat }} P_{\text {heat }}$ where $f_{\text {heat }}$ is a chosen (known) Gaussian function of $z$ which is a characteristic of the used actuator.

Due to the different orders of magnitude between $T$ and $\mathbf{b}$ and to the fact that only small variations of the magnetic field are considered, the dependence of the bootstrap current, the plasma resistivity and the thermal diffusion coefficient with the magnetic field may be neglected. This allows a linearization for the feedforward computation, using, for the computation of these quantities, the measurement of the magnetic field instead of the foreseen reference $\mathbf{b}_{\mathbf{d}}$. It has to be noticed however that doing so, the stabilization with the state feedback control will be obtained only locally, when the requested references will be close enough from the system initial state values.

Remark 5.1. One can remark that only the influence from the thermal domain to the electromagnetic one is represented and not the opposite sense. This choice however is justified by the assumption that the diffusion time-scales of each domain are separated by several orders of magnitude. The temperature establishes thousand times faster than the magnetic field does. In consequence, the magnetic field can be considered as "static" in the computation of the MHD couplings.

Finally, the feedforward is deduced from the steady state equation (5.3) using the relation between the safety factor $q$ and the magnetic state $\mathbf{b}$ in equation (4.2). The obtained feedforward $u_{d}$ is:

$$
\begin{aligned}
\left(\begin{array}{c}
P_{\text {ext }} \\
\left(P_{\text {heat }}\right)^{3 / 2} V_{\text {loop }} \\
P_{\text {heat }}
\end{array}\right)_{d}= & \left(\left(\begin{array}{c}
w^{f}\left(z_{1}\right) \\
w^{f} f\left(z_{2}\right) \\
w^{f}\left(z_{3}\right)
\end{array}\right) C\right)^{-1} \\
& \times\left[\begin{array}{c}
2 \pi\left(B_{\phi 0} a^{2}\right)\left(\frac{z_{1}}{q_{1 d}}, \frac{z_{2}}{q_{2 d}}, \frac{z_{3}}{q_{3 d}}\right)^{T} \\
+\left(\begin{array}{c}
w^{f}\left(z_{1}\right) \\
w_{f}^{f}\left(z_{2}\right) \\
w^{f}\left(z_{3}\right)
\end{array}\right)\left(J_{1} G_{m g}\right)^{-1} C_{\text {bs.d }} S_{\text {Joule }}
\end{array}\right]
\end{aligned}
$$

where $C=-\left(J_{1} G_{m g}\right)^{-1}\left(\begin{array}{ccc}f_{\text {ext }} & C_{R} J_{2}^{-1} J_{4} & C_{b s} f_{\text {heat }}\end{array}\right)$; $C_{R}=R /\left(P_{\text {heat }}\right)_{d}^{3 / 2}$, while $z_{1}, z_{2}, z_{3}$ are the three positions of the three references $\left(q_{1}, q_{2}, q_{3}\right)_{d}$ for the safety factor.

The feedforward control is thus derived from the steady state for the system obtained by the linearization at each time step of the non-linear parameters $R, R_{T}, G_{T}$ and $J_{b s}$. The feedback control is then required not only to increase the convergence speed but also to overcome the errors caused by the linearization assumptions.

\subsection{Controller tuning}

The interconnected system naturally converge to its equilibrium thanks to the two dissipations represented by the dissipation matrices $R$ and $R_{T}$. We decide to preserve the interconnection structure $\mathcal{J}$ of the original system, hence not modifying the internal dynamical couplings $\left(\mathcal{J}_{a}=0\right)$. Our control design consists in setting $\mathcal{R}_{a}$ constant such that $\mathcal{R}_{d}=\mathcal{R}_{d}^{T}>0$ (the desired dissipation rate) and then to determine the shaped Hamiltonian $\mathbb{H}_{d}$ and the feedback signal $\delta u$ with the help of the matching equation (4.6) and the integrability, equilibrium and stability conditions (2.6-2.8).

The robustness of the controller with respect to two kinds of uncertainties is studied. The first kind of uncertainties are those on the system dissipation $\mathcal{R}$ resulting from poor estimations of the plasma resistivity $\eta$ and the thermal diffusion coefficient $\chi$. The second kind of uncertainties are related to the linearization assumptions made in the derivation the feedforward control and in the approximation of the bootstrap current $\mathbf{J}_{b s}$.

Briefly, with a choice of supplementary dissipation $\mathcal{R}_{a}$ such that $\mathcal{R}_{d}$ is sufficiently large, we can handle these uncertainties (see subsection 3.3). Of course, the actuator power saturation will prevent us to compensate very large perturbations. Besides, the designed controller being basically a proportional controller, the choice of large values for the proportional gain may create undesired oscillations and instability for the closed loop system. In fact, the linearization condition in (3.3) isn't satisfied anymore.

\subsection{Simulation}

Three reference values for the safety factor $q$ are defined respectively at the radial relative coordinates $z=$ $0.1,0.3$, and 0.4 . The feedforward calculus gives $u_{d}$, the whole reference profile $q_{d}$ and the average temperature profile $T_{d}$, corresponding to these three references and taking into account the actuators constraints. The IDA-PBC control determines the feedback signal $\delta u$ from matching equation (2.4) to correct the error $X$. The IDA-PBC parameters are designed as discussed in the previous subsection, with the choice of $\mathcal{J}_{a}=0$ and $\mathcal{R}_{a}$ such as:

$$
\mathcal{R}_{a}=\left[\begin{array}{cc}
\left(\begin{array}{cc}
0 & 0 \\
0 & R_{a 1}
\end{array}\right) & 0 \\
0 & \left(\begin{array}{cc}
R_{a 2} & 0 \\
0 & 0
\end{array}\right)
\end{array}\right]
$$


where the positive diagonal matrix $R_{a 1}$ and $R_{a 2}$ account for the dampings added in electromagnetic domain and thermal domain respectively. As a particular case one can set only three diagonal values for the matrices $R_{a 1}$ and $R_{a 2}$ which correspond to three chosen reference positions.

The proposed IDA-PBC controller is tested on the RAPTOR code with the TCV configuration. The simulation results are showed as below.
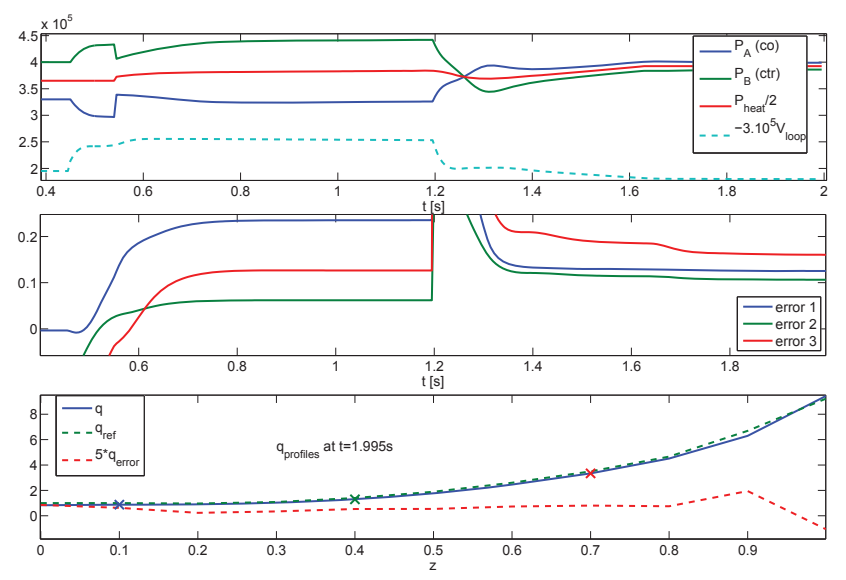

Figure 5.1: Feedforward control of coupled system
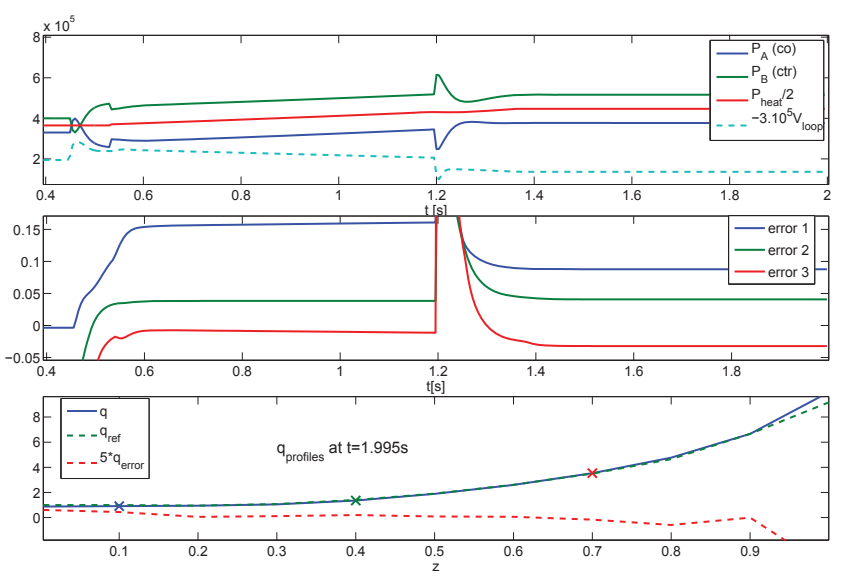

Figure 5.2: Feedback control of coupled system

Figures 5.1 and 5.2 show respectively the results obtained with the feedforward and the feedback controls. The heating actuator starts at $t=0.2 \mathrm{~s}$ with the initial values $\left(P_{\text {ext }}, V_{\text {loop }}, P_{\text {heat }}\right)_{\text {init }}=(40 \mathrm{~kW},-0.6 \mathrm{~V}, 300 \mathrm{~kW})$, whereas at $z=(0.1,0.3,0.7)$ the reference $q$-profile is set as $q_{a}=(0.85,1.1,2.4)$. Then at $t=0.8 s$, the reference is changed to $q_{b}=(1,1.4,3)$.

The feedforward does bring the $q$-profile close to the reference values but the actuator values as well as the $q$ profile oscillate around the equilibrium due to the parameter linearization and approximation. The feedback however makes effort to improve the result by continuing to react significantly on $P_{\text {heat }}$. However, it doesn't succeed in stabilizing the middle reference value for the $q$-profile. It
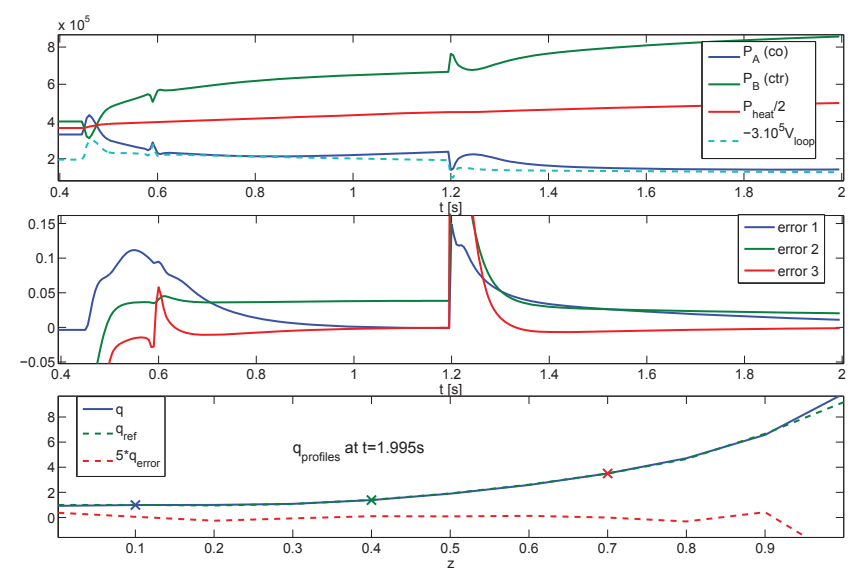

Figure 5.3: Feedback control of coupled system with a supplementary integrator

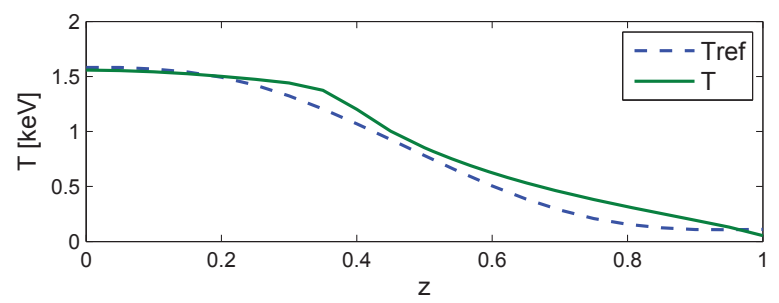

a.

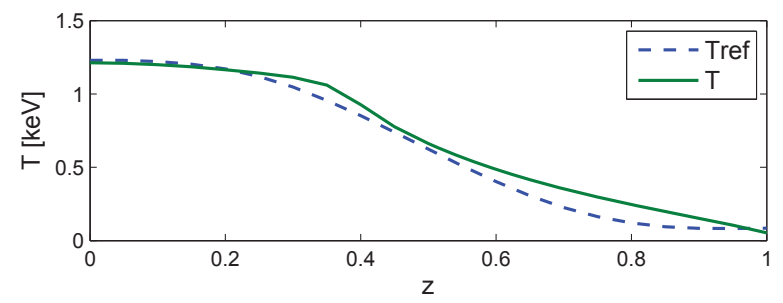

b.

Figure 5.4: $T$ profiles at $t=0.7 \mathrm{~s}(\mathrm{a})$ and $t=1.5 \mathrm{~s}(\mathrm{~b})$

is mainly regulated by $P_{\text {heat }}$, which continues to increase till the end. An integrator is implanted for the simulation presented in figure 5.3. A signification improvement is obtained for the first and the third points, which are directly affected by $P_{\text {ext }}$ and $V_{\text {loop }}$, while $P_{\text {heat }}$ makes impact indirectly to the second point via the TMHD couplings $J_{b s}$. The figure 5.4 shows the temperature profile vs the reference defined by the feedforward control at $t=0.7 \mathrm{~s}$ and $t=1.5 \mathrm{~s}$. Although these two profiles are built by two different discretization methods, they still match quite well to each other. On the other hand, these results lead to a discussion about the reference choice: should we take two reference points for the $q$-profile and one for the $T$ profile, the latter being directly affected by the heating source $S_{\text {heat }}$.

\section{Conclusion}

In this paper, an IDA-PBC feedback law is presented for the resistive diffusion of the magnetic flux and then for 
the coupled TMHD model - a set of two interconnected models for the resistive and thermal diffusions inside the tokamak plasma. The control methodology is based on IDA-PBC and the control law is derived from a PCH control model obtained from the geometric/ symplectic discretization of the corresponding coupled PDEs. An integrator is also successfully used in order to eliminate the static errors. The actuator constraints and limitations are taken into account in the equilibrium computation for the feedforward control action. The temperature profile and its influence on the resistivity coefficients are integrated into the control law via the Magneto-HydroDynammics couplings. The controllers have been tested in simulations with the RAPTOR code, and compared against the experimental data. A convergence has been observed with the computed feedforward and feedback controls. In future works, we can increase performance using simultaneous control of the thermal and magnetic models. Besides, the matching idea will be used to perform boundary and distributed (finite rank) feedback control directly on the infinite dimension port Hamiltonian model.

Beside the very efficient controller design, one of the difficulties of IDA-PBC is how to choose the parameters $\mathcal{J}_{d}, \mathcal{R}_{d}, \mathbb{H}_{d}$ regarding the system and actuator constraints. An optimal IDA-PBC control can be considered as one of the solutions for this problem, which is another objective of our future works.

\section{Acknowledgment}

The authors would like to thank S. BRÉMOND, O. SAUTER, H.B. LE and the TCV team of EPFL (École Polytechnique Fédérale de Lausanne), Switzerland for their support to the RAPTOR simulations and TCV experiments.

\section{References}

[1] F.B. Argomedo, C. Prieur, E. Witrant, and S. Brémond. A strict control lyapunov function for a diffusion equation with time-varying distributed coefficients. IEEE Transactions on Automatic Control (2012), 2012.

[2] M. Ariola and A. Pironti. Magnetic Control of Tokamak Plasmas. Advances in Industrial Control. Springer Verlag, London, 2008.

[3] J. Blum. Numerical Simulation and Optimal Control in Plasma Physics. Gauthier-Villars, 1989.

[4] M.D. Boyer, J. Barton, E. Schuster, and et al. First-principlesdriven model-based current profile control for the diii-d tokamak via lqi optimal control. Plasma Physics and Controlled Fusion, (55), 2013.

[5] F. Felici and O. Sauter. Non-linear model-based optimization of actuator trajectories for tokamak plasma profile control. Plasma Physics and Controlled Fusion, 54, 2012.

[6] F. Felici, O. Sauter, S. Coda, B.P. Duval, T.P. Goodman, J.M. Moret, J.I. Paley, and the TCV Team. Real-time physics-modelbased simulation of the current density profile in tokamak plasmas. Nuclear Fusion, 51, 2011.

[7] O. Gaye, E. Moulay, S. Brémond, L. Autrique, R. Nouailletas, and Y. Orlov. Sliding mode stabilization of the current profile in tokamak plasmas. Conference on Decision and Control (2011), Orlando, Florida, USA, December 2011.
[8] H. Khalil. Nonlinear Systems. Prentice Hall, Upper Saddle River, New Jersey, USA, 3rd edition, 2002.

[9] D. Moreau, F. Crisanti, X. Litaudon, and et al. Real-time control of the q-profile in jet for steady state advanced tokamak operation. Nuclear Fusion, 46:870-882, August 2003.

[10] D. Moreau and et al. A two-time-scale dynamic-model approach for magnetic and kinetic profile control in advanced tokamak scenarios on jet. Nuclear Fusion, 48(10):1-38, July 2008.

[11] R. Moulla, L. Lefèvre, and B. Maschke. Pseudo-spectral methods for the spatial symplectic reduction of open systems of conservation laws. Journal of Computational Physics, 231(4):1972-1992, December 2012.

[12] R. Ortega and E. Garcia-Canseco. Interconnection and damping assignment passivity-based control: A survey. Eur. J Control, 10:432-450, 2004.

[13] R. Ortega and Mark W. Spong. Stabilization of underactuated mechanical systems via interconnection and damping assignment. Automatic Control, 47:1218 - 1233, 2002.

[14] R. Ortega, A.J. van der Schaft, F. Castanos, and A. Astolfi. Control by interconnection and standard passivity-based control of port-hamiltonian systems. IEEE Trans. on Automatic Control, 53(11):2527-2542, 2008.

[15] R. Ortega, A.J. van der Schaft, B. Maschke, and G. Escobar. Interconnection and damping assignment: passivity-based control of port-controlled Hamiltonian systems. Automatica, 38(4):585-596, 2002.

[16] Y. Ou, C. Xu, and E. Schuster. Robust control design for the poloidal magnetic flux profile evolution in the presence of model uncertainties. IEEE Transactions on Plasma Sciences, 32(3):375-382, March 2010.

[17] Y. Ou, C. Xu, E. Schuster, T.C. Luce, J.R. Ferron, M.L. Walker, and D.A. Humphreys. Optimal tracking control of current profile in tokamaks. IEEE Transaction on Control Systems Technology, 19(2):432-441, March 2011.

[18] H. Ouarit, S. Brémond, R. Nouailletas, E. Witrant, and L. Autrique. Validation of plasma current profile model predictive control in tokamaks via simulations. SOFT Porto, Portugal (2010), 2010.

[19] A. Pironti and M. Walker. Fusion, tokamaks and plasma control. IEEE Control Systems Mag., 25:30-43, 2005.

[20] Trang.N.M. Vu, L. Lefèvre, R. Nouailletas, and S. Brémond. Geometric discretization for a plasma control model. IFAC Joint conference: 5th Symposium on System Structure and Control (2013); Grenoble, France.

[21] Trang.N.M. Vu and L.Lefèvre. Material balance and closure equations for plasmas in tokamaks. IFAC Workshop on Thermodynamic Foundations of Mathematical Systems Theory, July 13-16 2013.

[22] J. Wesson. Tokamaks. Third edition. Oxford Science Publications, 2004.

[23] E. Witrant, E. Joffrin, S. Brémond, G. Giruzzi, D. Mazon, O. Barana, and P. Moreau. A control-oriented model of the current profile on tokamak plasma. Plasma Physics and Controlled Fusion, 49:1075-1105, 2007. 